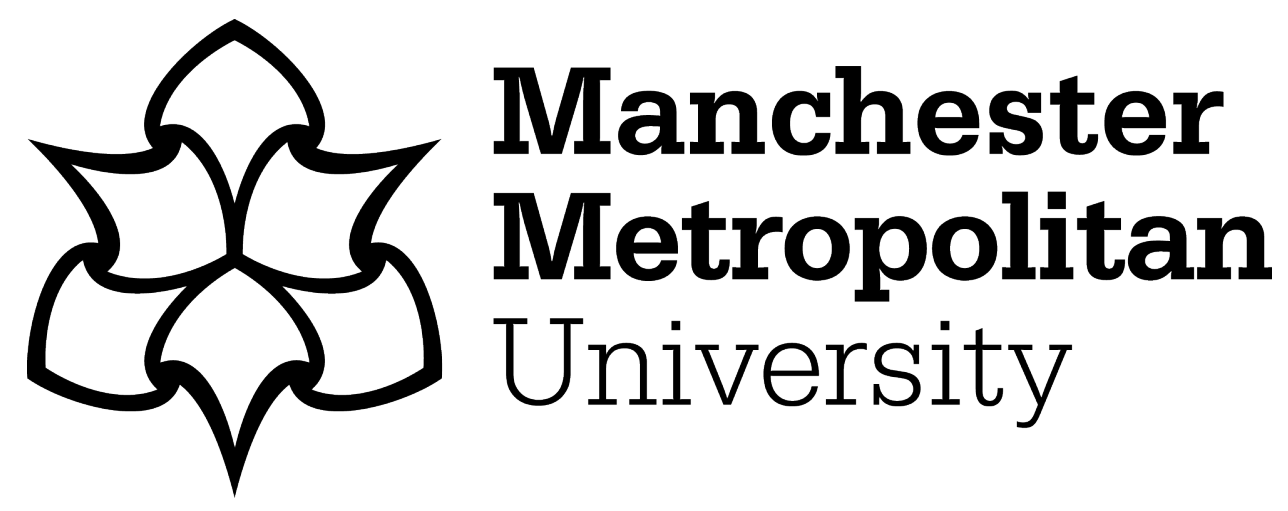

Melnyk, Paul, Djahel, Soufiene ORCID logoORCID: https://orcid.org/00000002-1286-7037 and Nait-Abdesselam, Farid (2020) Towards a Smart Parking Management System for Smart Cities. In: 5th IEEE International Smart Cities Conference (ISC2 2019), 14 October 2019 - 17 October 2019, Casablanca, Morocco.

Downloaded from: https://e-space.mmu.ac.uk/623879/

Version: Accepted Version

Publisher: IEEE

DOI: https://doi.org/10.1109/ISC246665.2019

Please cite the published version 


\title{
Towards a Smart Parking Management System for Smart Cities
}

\author{
Paul Melnyk ${ }^{\mp}$, Soufiene Djahel ${ }^{\mp}$ and Farid Nait-Abdesselam ${ }^{ \pm}$ \\ ${ }^{\mp}$ Department of Computing and Mathematics, Manchester Metropolitan University, UK \\ ${ }^{ \pm}$CSEE Department, University of Missouri Kansas City, USA \\ \{melnyk.paul@googlemail.com, s.djahel@mmu.ac.uk,naf@umkc.edu\}
}

\begin{abstract}
Smart parking management aims to leverage advanced sensing and monitoring technologies to optimise the usage of parking spots within large car parks and ease parking for drivers by directing them to available parking spots, thereby reducing unnecessary stress and delay that further worsen the traffic conditions. The idea of smart parking development began to make parking easier, faster, with less of a drain on resources which are slowly disappearing. The aim of the Smart Parking Management System (SPMS) proposed in this paper is to assist drivers in alleviating several issues linked to parking. Our SPMS provides a sensing platform supported by a mobile app to enable real-time interaction of drivers with the car park infrastructure in a way that minimises drivers' search time for an empty parking sport within large multi-storey car parks and facilitates the localisation of their parked cars. To validate the efficiency of our SPMS, a small scale test-bed was developed as a proof of concept and tested under five different scenarios. The obtained results highlight its effectiveness and call for its potential integration in real world car parks.
\end{abstract}

Keywords - Smart Parking Management, Sensors, ITS.

\section{INTRODUCTION}

Smart Cities are a current emerging trend that aims to effectively and smartly automate the monitoring, access and usage of the infrastructure underpinning the major services offered to the citizens. Advanced wireless sensing technologies, machine learning techniques, $5 \mathrm{G}$ networks and big data analytics tools are among the main enablers of secure and effective management of the often-limited city resources. If its usage is properly optimized such resources can significantly assist in revolutionizing the citizens life through improved education, better and more affordable healthcare and cheaper, greener and more comfortable transport.

The success of Intelligent Transportation Systems (ITS), that aim to bring drastic change to the transport sector to efficiently tackle the devastating impact of the increasing road traffic congestion, is reliant upon the real-time effective cooperation between the main components of any Traffic Management System (TMS) which are the routing, prediction and parking management systems, as stated in [1]. Such cooperation provides the traffic authorities with a powerful tool to control the flow of vehicles inside the city, optimize the usage of the available resources and prevent the creation of bottlenecks at major critical locations. Moreover, the Mobility as a Service (MaaS) paradigm will require this cooperation to effectively manage the fleet of smart vehicles offering the mobility service to citizens.
Despite the above innovation in transport, the Parking Management Systems (PMS) have not seen tremendous advances in the way the availability of parking spots is being advertised to drivers and the tools made available to them to easily park and locate their vehicles within large multi-storey car parks. PMSs' inefficiency is highlighted often, as drivers spend huge amount of time searching for free spots; even though on average only around $80 \%$ of spots are occupied [2]. It has been shown by INRIX that the average UK driver spends around 44 hours a year searching for parking, equating to $£ 733$, totalling $£ 23.3$ billion for the country [3]. In this study, London ranked as the worst city to park in with an annual search time of 67 hours or 12 minutes per journey. Moreover, as analysed by Admiral; $64 \%$ of drivers feel stress when parking, and ' $71 \%$ stating there were not enough parking spaces [4]. Therefore, it is necessary to modernize the existing PMSs by making them smart through the usage of advanced ICT driven solutions to ultimately reduce the anxiety, stress and time wasted due to parking.

The remainder of the paper is organised as follows. Section II briefly discusses the literature followed by a description of the key principle of our proposed SPMS in Section III. In Section IV, we present our test-bed and discuss the evaluation scenarios and results. Finally, in Section V, we conclude the paper and present our future work plan.

\section{CRITICAL ANALYSIS OF EXISTING SPMSS}

In this section we will briefly discuss a selection of the most important recent attempts on designing SPMSs.

In [5], an IoT (Internet of Things) based E-parking system was developed in order to solve some issues within parking structures. The key features of the proposed system range across a few components. The parking lot monitoring component regularly checks the state of the parking spots based on time differences between sending and receiving a signal. Once a change is detected a photo is taken and sent to the parking management system. If a car is improperly parked, the system fails to extract a licence plate number and therefore a warning is issued. In this system, reservations are made through the application available on users' mobile devices. This system is effective in detecting incorrectly parked cars and ensures up to date information by sending waves once every 60 milliseconds. However, it does not provide an efficient way to quickly locate 
free parking spots, which is an important feature especially needed in large multi-storey car parks.

Mejri et al. proposed a parking reservation system called "ROSAP" in [6] to help drivers find a free spot closer to their final destination. ROSAP aims to achieve a reasonable walking distance between the chosen parking spot and the destination, not exceeding $400 \mathrm{~m}$ in average. The key novelty in this work is the development of a generic solution for parking as opposed to other scenario-specific systems developed in the past. The main sound feature of ROSAP is the reduction of the walking distance from the parking spot to the driver destination as well the easiness of reservation as shown in the evaluation results. However,the main drawbacks of ROSAP consists in its associated privacy issues as drivers might be reluctant to disclose their final destination in addition to its quite slow response to drivers' requests $(1 \mathrm{~min})$.

Optimizing the usage of car parks was investigated in [7] in order to avoid under-utilizing the available parking infrastructure in mega cities, which may in turn extend drivers search time for available parking spots. This has been proven to significantly contribute to increased congestion on the roads. In this work, a new data model is proposed to speed up and ease free parking spots search within a large parking structure. This allows drivers to not only find the right entry to the parking structure but also to find specific parking spaces within the lot. Although this system features reliable sensor reading and monitoring of the occupancy of parking spots, its accuracy is questionable as occupancy information is updated every 5 minutes which may prohibit real-time access to accurate parking availability information.

Creating an effective parking solution in which the state of the space (free or occupied) is displayed using a light was proposed in [8] by leveraging sensors and light coding scheme. In this scheme, a blue light means occupied spot; a red light means incorrectly filled spot and a yellow light means an empty spot. This work is different from the aforementioned ones as it offers two different types of parking - economy and business. The main difference between these being their location within the parking structure and the price; business spots being closer to the exit and their price is higher. Using lights to display the state of parking spots could be effective if the car to be parked is in the same level (floor) where the the free spot is located, however in multi-storey car parks the driver might waste an additional time before reaching the floor where the yellow light is on, thus affecting the overall efficiency of the parking system. Moreover, the drivers must have the light linked to the available spot in their vision, which can be especially difficult in car parks where there are larger vehicles blocking the view or pillars between levels.

In the light of the above discussion we will propose in this paper an SPMS that facilitates the localisation of free spots within car parks as well as quickly directing drivers to the location of their parked vehicles.

\section{SPMS DESIGN PRINCIPLES}

Smart Parking Management Systems are a promising solution to one of the key issues within smart cities; the inefficient management of parking spots. The key objective of an SPMS is to efficiently allocate available parking spots to drivers while minimising their search time and provide them with a smart and efficient way for easily locating their parked cars, especially in large multi-storey parking structure, and finally paying the parking fee. SPMSs usually rely on data collected from the deployed monitoring equipment such as RFID tags and sensors to acquire accurate view on the occupancy level of the car park and the exact location of the available spots, if the technology that enables that is in place.

IoT plays a major role in the success of SPMSs. Indeed, the advancement of the IoT has led to significant changes in parking management as it allows for easy expansion without the need to run cables and networking devices to connect new spaces/structures; sensors, cameras, barriers and so on can be connected wirelessly to the monitoring system in place. IoT devices are already widely deployed to serve the needs of various application domains in smart cities, ranging from lighting to surveillance cameras etc., and with the development of SPMSs the IoT's application scope could be further expanded to collect information about points of interest visited by drivers after parking; allowing businesses to capitalise upon this knowledge.

Figure 1 shows the architecture of the proposed SPMS and the interactions between its different components. In this architecture, SQL databases are connected to a servlet, allowing data to be sent and retrieved. The servlet is controlled from a code where data is sent through Message Queuing Telemetry Transport (MQTT) protocol or sent to the Application/Controller. MQTT protocol is a key component of our architecture as it collects and processes data as necessary. This data can also be used to move servo motors to open/close the car park barrier when the conditions are met.

The collected data from the car park infrastructure and the cars will be stored in a database, accessed through a JDBC controller and an Apache Tomcat server. Examples of such data include the following:

- Barrier Information - Serial numbers, location, entry or exit, etc.

- Spot Information - Sensor serial numbers, location, state, etc.

- Accessor (i.e., the car) Information - Current state, unique identifier, spot where it is parked (if applicable).

Notice that all parking spots and RFID detectors are controlled through Phidget sensors, paired with JAVA to process changes and send appropriate information where needed once changes are detected. The car park barriers, used in our prototype, are also controlled through Phidget, and will be moved (open or close) when the correct information is sent to the controller. The mobile application will be used to display information to the user depending on their current position/state within the car park. Through this mobile app 


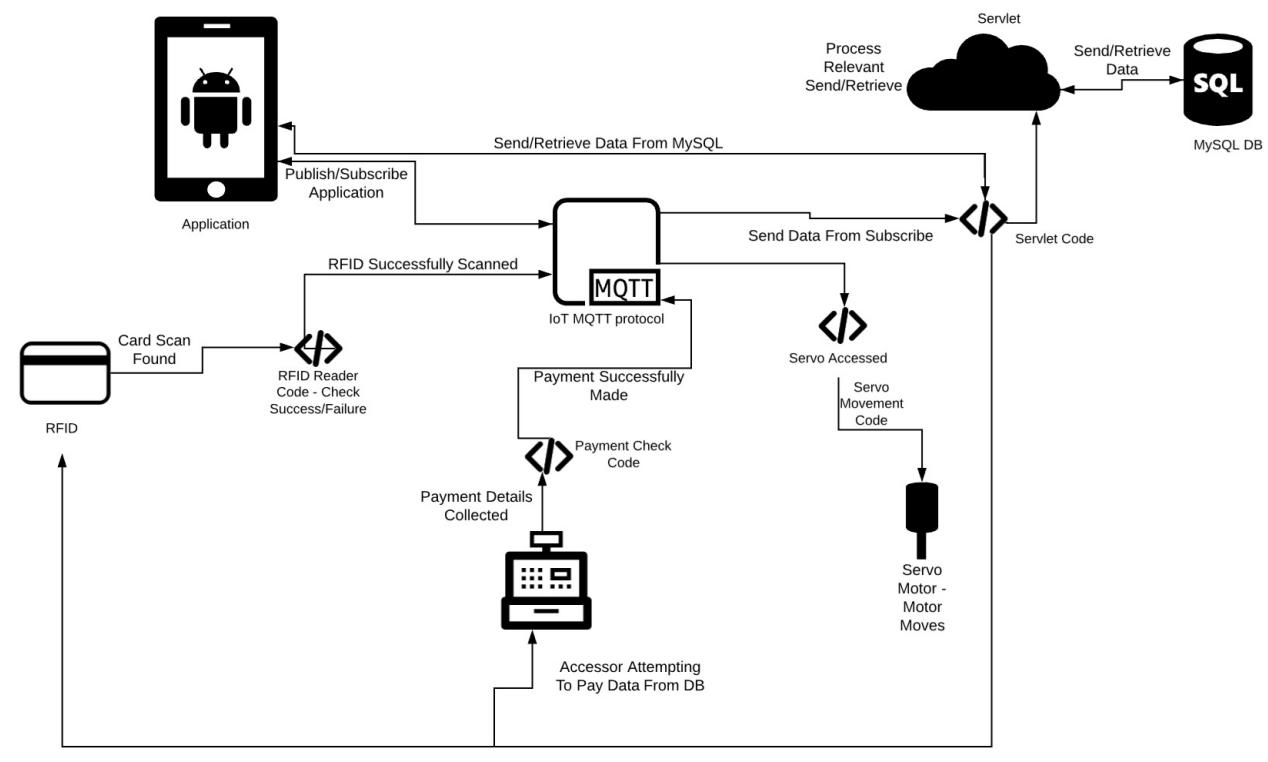

Fig. 1. The proposed SPMS architecture

the user will be able to enter/exit the car park, find the nearest available spot within the car park, view spot information for their parked vehicle and finally pay the parking fee.

\section{SPMS EVALUATION}

In this section we will briefly describe the hardware components used to set up our testbed as well as the scenarios tested to validate the effectiveness of our proposed SPMS.

\section{A. Hardware configuration}

The proposed SPMS was implemented on a small-scale testing bench using Phidget hardware as shown in Figure 2. The testbed consists of the following hardware components: two RFID Readers, two Servo Motors, six IR Reflective Sensors and one Interface Kit 8/8/8. The RFID readers are used to control the entry and exit gates when the application is unused and the servo motors act as the entry/exit gate. IR reflective sensors act as in-spot sensors that detect the state of the spot (free or occupied by a parked car), with all the IR sensors connected to a single Interface kit. Each major component of our SPMS (i.e., RFID, Servos and interface kit) is directly connected to a laptop acting as a server that collects information about the car park infrastructure and occupancy.

\section{B. Testing Scenarios}

1) RFID incorrect scanner: Accessors (i.e., cars or their drivers) with RFID cards are always able to scan both entry and exit scanners, meaning that the state of the accessor must be checked upon scanning; preventing users currently in the car park from accessing the entry barrier and vice versa. This function represents an important feature as it prevents users from accessing incorrect gates; preventing issues with the closest spot recommender, and does not require any direct user input. The result of an attempted gate access while the

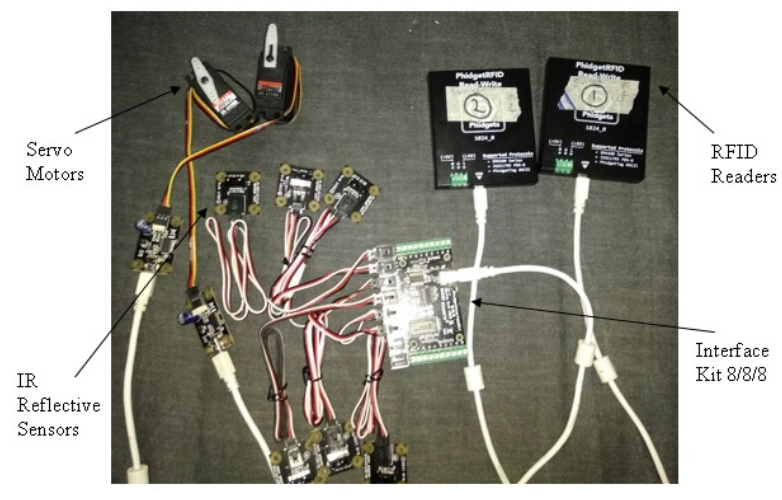

Fig. 2. Small scale testbed used in the evaluation of our SPMS

Fig. 3. RFID console output upon incorrect scanner access attempt

car is in an incorrect state (e.g., attempting to enter the car park through teh exit gate) is shown in Figure 3.

2) Attempting access when car park is full: Both RFID Management system and the mobile app have a functionality to prevent over population in the car park. When an accessor attempts entry, the server gathers the total number of available parking spots, which is then compared to the current accessors within the structure, either parked or searching for a vacant spot. In situations where these counts match, user access is rejected as no vacant spots will be available. Figure 4 displays how this entry rejection is viewed by the driver. This feature helps in avoiding the formation of queues inside the car park as it prevents drivers searching for vacant spots that are not present from accessing the car park.

3) Automatic spots assignment: When there is a number of accessors inside the car park the spots allocation process 


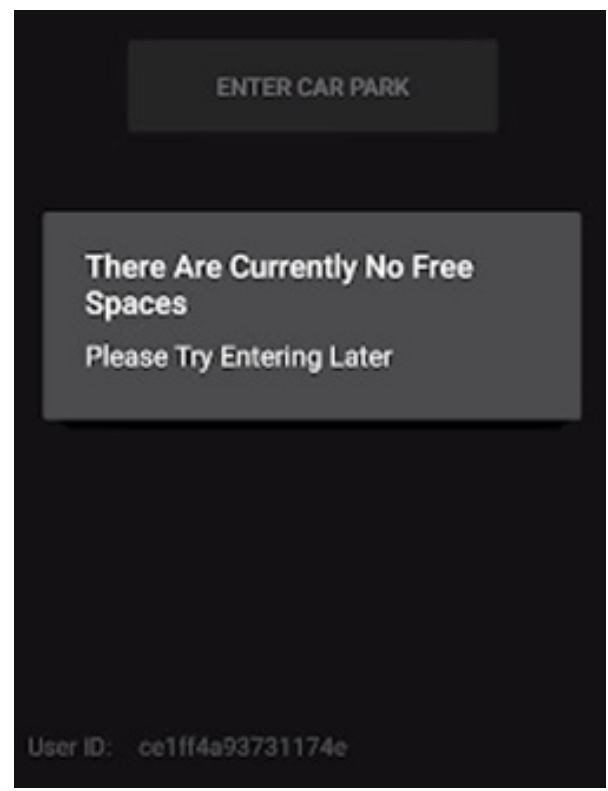

Fig. 4. Android application display upon an attempted access to the car park while it is ful

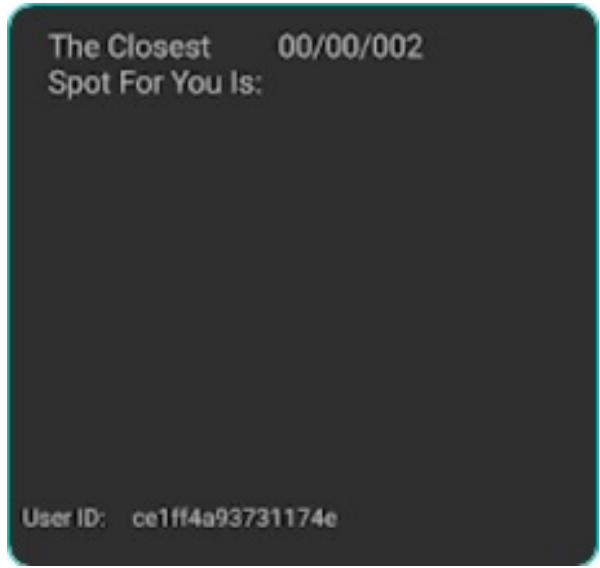

Fig. 5. Application output while the driver is looking for a free spot inside the car park

is triggered whenever a spot state changes to free (i.e., unallocated spot which is not linked to any existing user). If the number of accessors is equal to 1 then the spot is automatically assigned to the user and the information is updated in the database. This process is efficient as it reduces user input, thereby increasing usability with an elegant solution - leading to $70 \%$ average reduction in time searching as shown in the conducted survey by a number of volunteer users. If the number of accessors inside the car park is greater than 1 then the application follows the process described in IV-B4. A snapshot of the database update is shown in Figure 6. Figure 7 depicts the output of the mobile app displayed to the driver of the blue car parked in the spot 00/00/012 .

4) Multiple users spot assignment: As stated above, this scenario is met when a spot state changes and more than one accessor has met ready to park criteria (i.e., they are inside the car park looking for the closest available spot to park in). When this happens, an ArrayList is populated with all populated spots with un-allocated state, used to render a ListView within all applications in the ready to park state. Dynamic updates applied in our SPMS ensures that real-time and accurate spots availability information ids available to drivers; giving them the ability to choose their nearest spot and bind it to their account. If our SPMS is deployed in a largerscale real life situation this would be replaced with a GPS function, however on a small scale is unmanageable due to the minuscule differences in vehicle location. This also feeds back to IV-B3, if after user selection only one spot and one accessor remain to be allocated; automatic spot allocation occurs.

\section{Real-time updates}

The proposed SPMS works well to combat issues currently shown in prior SPMS proposals. As accessors are often unaware of where they wish to park our SPMS guides vehicles within the car park to the closest available spots ( it is not a reservation-based spots allocation but instead the allocation is made in real-time). On-screen updates were key priority tackled by the development of our SPMS to prevent issues where multiple users attempt to park within the same spot boundaries; leading to a queue that further affects the congestion in relation to parking. The SPMS tackled this with the usage of MQTT protocol, therefore, database and application updates were not related to a predetermined time count; but to a publish method triggered through reading changes from spot sensors, therefore always up to date. When comparing this SPMS to the work proposed by Silar et. al in [7] where updates occur once every 5 minutes, our SPMS results in a time saving of up to 4 minutes and 30 seconds at some occurrences (entering spot, payment processing, etc.), which has the potential to occur multiple times per accessor cycle. If this time saving occurs as little as 10 times per day a total of 45 minutes would be saved; adding up to an average difference of 21 hours a month, showing huge success for real-time updates.

\section{Finding the closest spot}

Finding the closest spot to an accessor is key to the success of the proposed SPMS in order to efficiently process the spots allocation requests. By using the barrier accessed location and the spot sensor location coded as $\left(\right.$ floor $_{i d}$, row $_{i d}$ spot $_{i d}$ ) finding the best spot for a given accessor is a straightforward process. An example of the output displayed to the driver running our mobile app is shown in Figure 5 where the closest spot is the one with the identifier 00/00/002 referring to the spot number 12 or the row 0 located in the ground floor of a multi-storey car park. In a real world multi-storey car park the processing time for a larger number of spots is negligible due to the existing powerful computing tools.

A demo of our SPMS can be found here: https://youtu.be/PRg2JWwcE1Y

\section{CONClusion}

In order to alleviate the stress and anxiety experienced by the drivers while searching for an available parking spot 


\begin{tabular}{|c|c|c|c|c|c|c|}
\hline & tagid & inside_carpark & mobile_access & spot_parked & last_accessed_barrier_serial & enter_spot_date_time \\
\hline \multirow[t]{11}{*}{ • } & ce $1 \mathrm{ff} 4 \mathrm{a} 93731174 \mathrm{e}$ & 1 & 1 & 3147441 & 305689 & $2019 / 03 / 2506: 11: 23$ \\
\hline & $01077594 b 6$ & 0 & 0 & 0 & 306409 & N/A \\
\hline & $014773897 \mathrm{~b}$ & 0 & 0 & 0 & 306409 & $\mathrm{~N} / \mathrm{A}$ \\
\hline & 14041241 & 0 & 0 & 0 & 306409 & N/A \\
\hline & $21 c 35441 c f d 3 a e 38$ & 0 & 1 & 0 & 306409 & N/A \\
\hline & 3454 ffoe $7503 b 24$ & 0 & 1 & 0 & 306409 & N/A \\
\hline & $3 \mathrm{~d} 10 \mathrm{a} 265370 \mathrm{c} 03 \mathrm{fg}$ & 0 & 1 & 0 & 306409 & N/A \\
\hline & $6 c 13986 \mathrm{~d} 50 \mathrm{ec} 8 \mathrm{e} 16$ & 0 & 1 & 0 & 306409 & N/A \\
\hline & $9611 a 852325 d 97 b a$ & 0 & 1 & 0 & 306409 & N/A \\
\hline & $\mathrm{f} 4 \mathrm{~d} 3216 \mathrm{~d} 70 \mathrm{c} 3643 \mathrm{~b}$ & 0 & 1 & 0 & 306409 & N/A \\
\hline & f7e0c0951d91e011 & 0 & 1 & 0 & 306409 & N/A \\
\hline
\end{tabular}

Fig. 6. Automatic spot assignment database update

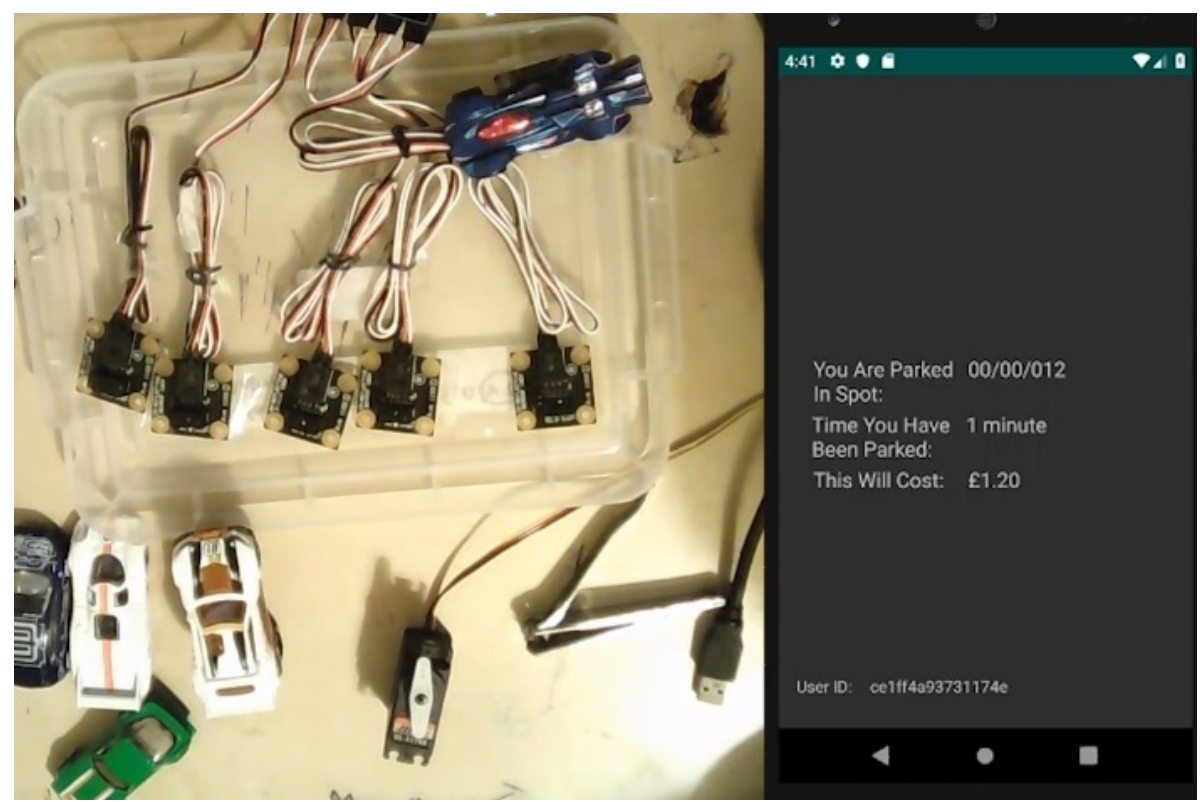

Fig. 7. Application output while the blue vehicle is parked in the spot with the ID 00/00/012

and minimize their search time, we proposed a new Smart Parking Management System (SPMS) that provides real-time information about availabilities of parking spots to drivers and assists them is easily finding their parked cars, especially in large car parks. The effectiveness of our SPMS has been validated through a small scale test-bed, where a number of scenarios have been tested. In the future, if access is granted to real-world car parks, our test-bed could be extended to undertake a pilot test with real cars and assess its effectiveness under real conditions.

\section{REFERENCES}

[1] S. Djahel et al. A communications-oriented perspective on traffic management systems for smart cities: Challenges and innovative approaches. IEEE Communications Surveys Tutorials, 17(1):125-151, Firstquarter 2015.

[2] British Parking Association; Skyblue Research.

[3] INRIX. Searching for parking costs the uk $£ 23.3$ billion a year. In http://inrix.com/press-releases/parking-pain-uk/, July 2017.

[4] C. Frampton. Average driver spends nearly two days a year searching for parking. In https://www.admiral.com/magazine/news-and-currentaffairs/drivers-spend-fourty-four-hours-a-year-searching-for-a-parkingspace, July 2017.
[5] P. Sadhukhan. An iot-based e-parking system for smart cities. In 2017 International Conference on Advances in Computing, Communications and Informatics (ICACCI), pages 1062-1066, Sep. 2017.

[6] N. Mejri et al. Reservation-based multi-objective smart parking approach for smart cities. In 2016 IEEE International Smart Cities Conference (ISC2), pages 1-6, Sep. 2016.

[7] J. Silar et al. Smart parking in the smart city application. In 2018 Smart City Symposium Prague (SCSP), pages 1-5, May 2018.

[8] G. Yan et al. Smartparking: A secure and intelligent parking system. IEEE Intelligent Transportation Systems Magazine, 3(1):18-30, Spring 2011. 